\title{
PERFIL EPIDEMIOLÓGICO DOS PACIENTES PORTADORES DE CARCINOMA EPIDERMÓIDE COM DIAGNÓSTICO HISTOPATOLÓGICO ESTABELECIDO NO CENTRO DE REFERÊNCIA DE LESÕES BUCAIS DA UNIVERSIDADE ESTADUAL DE FEIRA DE SANTANA NO PERÍODO DE 2006-015 .Lorena Araujo Almeida ${ }^{1}$; Gabriel Levi da Silva Cunha ${ }^{2}$; Rafael Cabral de Albuquerque Souza ${ }^{3}$ e Tarsila de Carvalho Freitas Ramos ${ }^{4}$ \\ 1.Bolsista PROBIC/UEFS, Graduando em odontologia, Universidade Estadual de Feira de Santana, email: loreaaraujo@gmail.com \\ 2. Participante do projeto, Graduando em odontologia, Universidade Estadual de Feira de Santana, email: gabriellscunha@gmail.com \\ 3. Participante do projeto, Graduando em odontologia, Universidade Estadual de Feira de Santana, email: rafael_cabral27@live.com \\ 4. Orientador, Departamento de Saúde, Universidade Estadual de Feira de Santana, email: tarsilafreitas@yahoo.com.br
}

PALAVRAS-CHAVE: Patologia oral, lesões orais, epidemiologia

\section{INTRODUÇÃO}

Entre as neoplasias malignas que acometem as estruturas bucais, o carcinoma epidermóide é a mais prevalente afetando predominantemente o gênero masculino. As faixas etárias mais prevalentes correspondem a sexta e sétima décadas de vida. A média de idade para o gênero masculino foi de 58,2 anos e para o feminino de 60,5 anos (ANJOS HORA et al., 2003). Segundo estudos de Anjos Hora (2003), as localizações anatômicas de maior prevalência para o Câncer Bucal foram a língua e o lábio, em ambos os sexos.

A pesquisa sobre Perfil epidemiológico dos pacientes portadores de Carcinoma Epidermóide (CEC) com diagnóstico estabelecido no Centro de Referência de Lesões Bucais (CRLB) da Universidade Estadual de Feira de Santana(UEFS) no período de 2007 a 2015 se propõe a investigar o perfil clínico e epidemiológico dos pacientes portadores de Carcinoma Epidermóide na população do semiárido baiano e os fatores de risco aos quais a mesma está exposta. O Câncer bucal se configura como um problema de saúde pública com impactos mundiais e através desta pesquisa poderemos conhecer o perfil da população acometida por esta patologia, tornando estas informações conhecidas aos cirurgiões dentistas. Os dados epidemiológicos encontrados servirão de base para a criação de políticas de prevenção e estratégias de ação visando o diagnóstico precoce desta patologia maligna.

\section{MATERIAL E MÉTODOS OU METODOLOGIA (ou equivalente)}

Foi realizado um estudo de corte seccional onde se verificaram todos os prontuários dos pacientes que possuíam resultado de Carcinoma Epidermóide em laudo de exame histopatológico no Centro de Referência de Lesões Bucais (CRLB) da Universidade Estadual de Feira de Santana/Bahia, no período de 2006 a 2015. Os dados foram coletados através de um formulário específico contendo informações sobre sexo, idade, cor, suspeita clínica, diagnóstico histopatológico, localização, características clínicas da lesão, consumo de tabaco, de álcool, de álcool e tabaco juntos, abandono do consumo de tabaco e álcool e presença de lesão associada a prótese.

Os prontuários dos pacientes atendidos no CRLB contêm o TCLE, onde é esclarecido que todas as informações contidas nos mesmos poderão ser utilizadas em pesquisas e divulgações científicas. Desta forma, o uso dos prontuários não causa desconforto ou constrangimento aos participantes do estudo uma vez que, com o intuito de minimizá-los, a participação destes é voluntária e o anonimato resguardado, garantindo o sigilo dos dados.

Os dados foram digitados e analisados utilizando o programa Statistical Package for Social Science - SPSS, versão 10.0, sendo apresentadas distribuições absolutas e percentuais. Após essa análise as informações foram sistematizadas em tabelas e gráficos com auxílio do 
Programa Excel da MICROSOFT CORPORATION (2003), seguindo a seqüência e distribuição das variáveis do estudo.

Vale ressaltar que este estudo é parte de um projeto que se encontra registrado no Comitê de Ética da Universidade Estadual de Feira de Santana (CEP-UEFS) sob protocolo $\mathrm{N}^{\circ}$ 015/2008, CAAE 0015.0.059.000-08.

\section{RESULTADOS E/OU DISCUSSÃO}

Foram encontrados 104 prontuários de pacientes diagnosticados com CEC. O perfil encontrado neste estudo está de acordo com outras pesquisas encontradas na literatura. Com relação ao gênero houve uma predileção pelo masculino, isso pode ser devido a uma maior exposição destes indivíduos a fatores de risco como álcool, tabaco e a exposição solar(Tabela 1) (OLIVEIRA; SILVA; ZUCOLOTO, 2006)' (BRENER et al., 2007)'(ABDO; GARROCHO; AGUIAR, 2002).

A idade avançada, incluindo pacientes acima de 60 anos, predominou nesta pesquisa, corroborando com outros estudos citados na literatura(OLIVEIRA; SILVA; ZUCOLOTO, 2006) '(WERNER; FONTANELLA, 2009). Os pacientes, em sua maioria, eram faiodermas, refletindo a população miscigenada do nordeste, esse é um dado sócio demográfico que muda de acordo com a região estudada, como demonstra algumas pesquisas com resultados de carcinomas bucais mais prevalentes em pacientes leucodermas e outras em melanodermas (Tabela 1) (WERNER; FONTANELLA, 2009)'(PEREZ et al., 2007).

Outras duas variáveis investigadas neste estudo foram o consumo do álcool e tabaco, os quais são dois fatores de risco para o CEC bem discutidos (OLIVEIRA; SILVA; ZUCOLOTO, 2006)' (BRENER et al., 2007)'(ABDO; GARROCHO; AGUIAR, 2002). Os pacientes em sua grande maioria, nesta pesquisa, faziam o uso combinado destes. Os usuários de álcool perfaziam $5 \%$ da amostra $(n=5)$, os que usavam apenas tabaco $19,7 \%(n=20)$ e os que faziam uso associado representavam a maioria, $70,3 \%$ da amostra $(n=71)$, o que aumenta ainda mais a possibilidade do desenvolvimento do câncer. Apenas $5 \%$ da população estudada nunca fez uso do tabaco e álcool(Tabela1).

As lesões investigadas nesta pesquisa, clinicamente, apresentaram o padrão mais descrito pela literatura (NEVILLE, 2009)'(WERNER; FONTANELLA, 2009): consistência dura, ulceradas, coloração vermelha, superfície rugosa, profundidade submucosa, implantação séssil (Tabela 2). A localização mais prevalente foi borda lateral de língua 25,5\% ( $\mathrm{n}=26)$, seguida de assoalho de boca 19,6\% ( $\mathrm{n}=20)$ e lábio inferior 13,7\% ( $\mathrm{n}=14)$ (Figura 1). Muitos estudos não apresentam alta incidência em lábio inferior. Nesta pesquisa, esse achado se deve ao fato da população nordestina se expor cronicamente ao sol e não fazer o uso adequado dos fatores de proteção(ABDO; GARROCHO; AGUIAR, 2002).(WERNER; FONTANELLA, 2009)'(PEREZ et al., 2007). O tipo de biópsia de escolha predominante foi a incisional e a maioria das lesões possuíram um desenvolvimento lento, com cerca de 12 meses de evolução (Tabela 2).

Tabela 1- Características sociodemográficas dos pacientes acometidos por CEC diagnosticados no Centro de Referência de Lesões Bucais (CLRB) da UEFS entre 2007 à 2015.

\begin{tabular}{lrr}
\hline Sexo & $\mathrm{n}$ & $\%$ \\
Feminino & 31 & 29,8 \\
Masculino & 73 & 70,2 \\
Cor da pele & & \\
Faioderma & 35 & 38,9 \\
Leucoderma & 23 & 25,6 \\
Melanoderma & 30 & 33,3 \\
Xantoderma & 2 & 2,2 \\
Idade & & \\
Até 60 anos & 51 & 49,0 \\
\hline
\end{tabular}




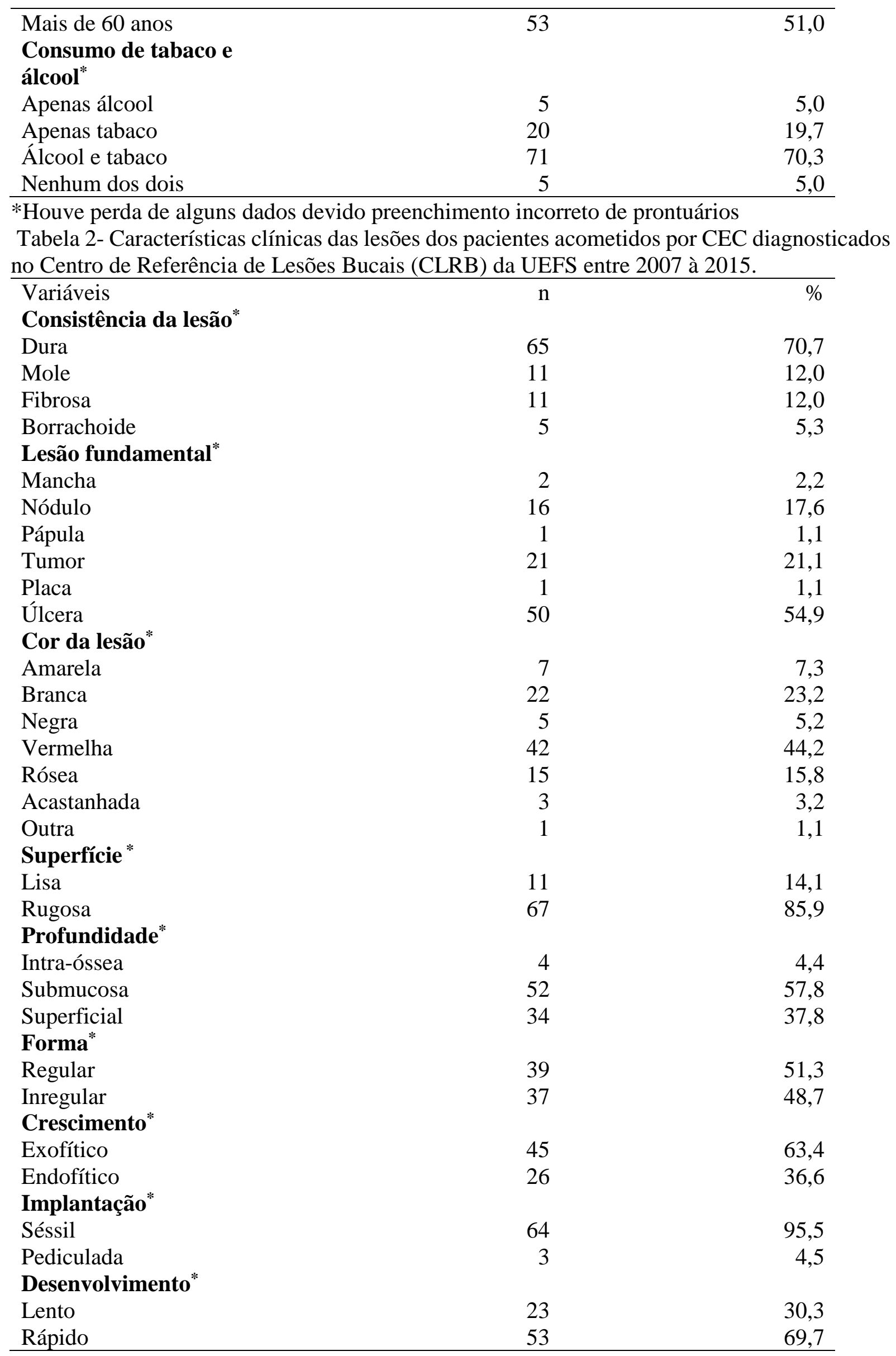




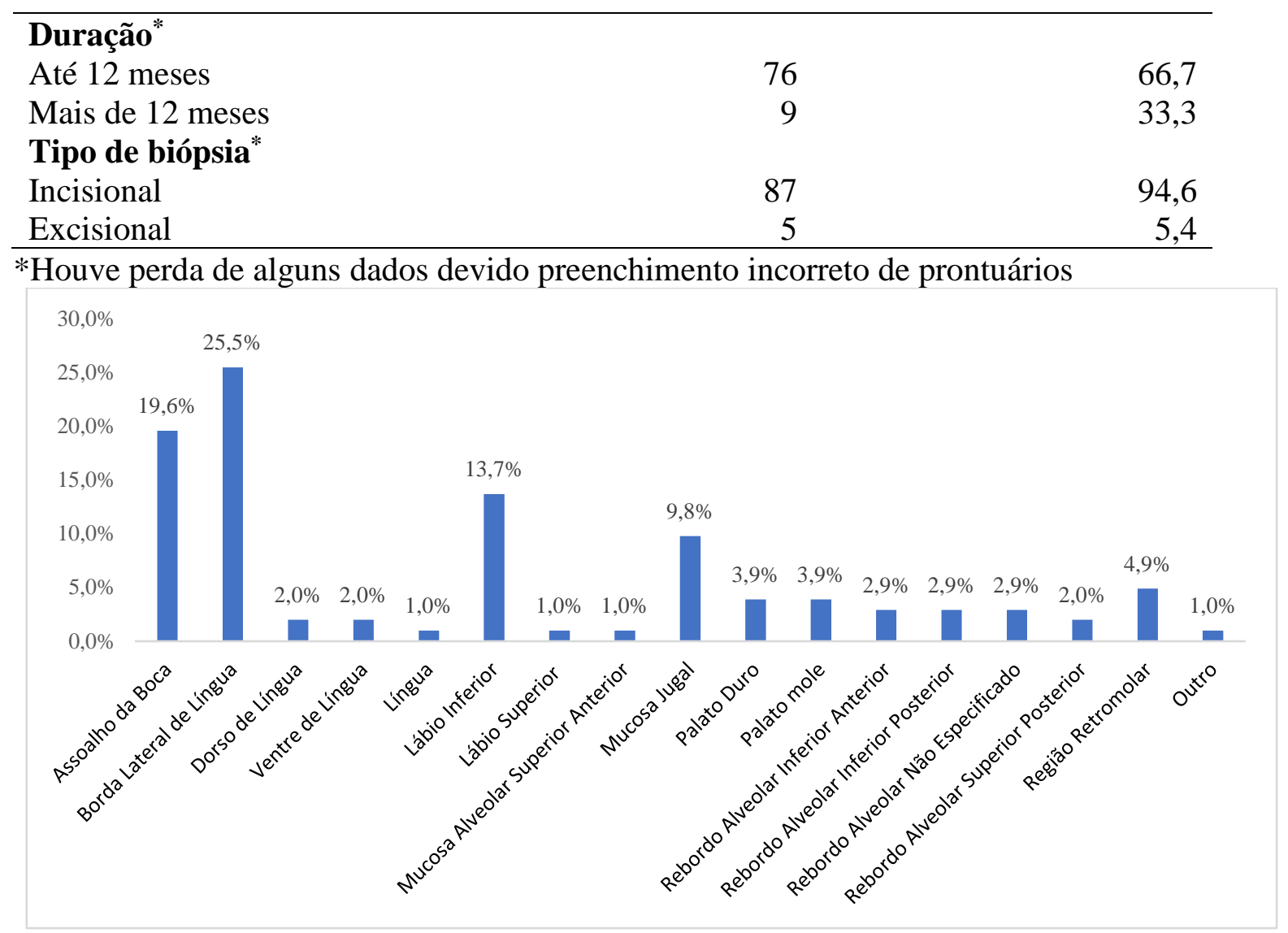

Figura 1: Localização anatômica das lesões dos pacientes acometidos por CEC diagnosticados no Centro de Referência de Lesões Bucais (CLRB) da UEFS entre 2007 à 2015

\section{CONSIDERAÇÕES FINAIS}

Os resultados desta pesquisa permitiram inferir que na população do Semiárido baiano atendida no CRLB, a maioria dos portadores de CEC são homens entre 51 e 70 anos, expostos aos fatores de risco, tabaco e álcool. As lesões foram predominantemente ulceradas, localizadas em língua, com coloração vermelha e superfície rugosa.

\section{REFERÊNCIAS}

ABDO, E. N.; GARROCHO, A. DE A.; AGUIAR, M. C. F. DE. Perfil do paciente portador de carcinoma epidermóide da cavidade bucal, em tratamento no Hospital Mário Penna em Belo Horizonte. Rev Bras Cancerol, v. 48, n. 3, p. 357-362, 2002.

ANJOS HORA, I. A. DOS et al. Estudo epidemiológico do carcinoma epidermóide de boca no estado de Sergipe. Ciência Odontológica Brasileira, v. 6, n. 2, p. 41-48, 2003.

BRENER, S. et al. Carcinoma de células escamosas bucal : uma revisão de literatura entre o perfil do paciente, estadiamento clínico e tratamento proposto. Revista Brasileira de Cancerologia, v. 53, n. 1, p. 63-69, 2007.

MELO, A. R.; PIRES, S. M. S.; RIBEIRO, C. F.; JUNIOR, R. L. C. A.; MELO, A. U. C. Prevalência de lesões bucais diagnosticadas no laboratório de patologia bucal da Universidade Tiradentes (2002- 2010). Revista de Cirurgia e Traumatologia Buco-maxilo-facial ISSN 1808-5210, 2014. NEVILLE, B. W. ET. AL. Patologia oral \& maxilofacial. Elsevier ed. Rio de Janeiro: [s.n.]. OLIVEIRA, L. R.; SILVA, A. R.; ZUCOLOTO, S. Perfil da incidência e da sobrevida de pacientes com carcinoma epidermóide oral em uma população brasileira. Jornal Brasileiro de Patologia e Medicina Laboratorial, v. 42, n. 5, p. 385-392, 2006.

PEREZ, R. S. et al. Estudo Epidemiológico do Carcinoma Espinocelular da Boca e Orofaringe. International Archives of Otorhinolaryngology, v. 11, n. 3, p. 271-277, 2007. WERNER, E.; FONTANELLA, V. Per fi I epidemiológico dos pacientes portadores de câncer bucal atendidos no Hospital Santa Rita, Porto Alegre / RS. Stomatos, v. 15, n. 28, p. 3-16, 2009. 